\title{
The Influence of Electric Dipoles on Bubble Coalescence
}

\author{
P. C. DuinevelD ${ }^{1}$ \\ J. M. Burgers Centre for Fluid Mechanics, Department of Applied Physics, University of Twente, P.O. Box 217, 7500 AE Enschede, The Netherlands
}

Received November 15, 1995; accepted March 13, 1996

\begin{abstract}
The influence of electric dipole moments of nonionic surfactants on inertia-dominated film thinning between two bubbles has been calculated. The repelling force due to dipole moments cannot be the cause of coalescence inhibition, as was suggested by $\mathbf{G}$. Keitel and U. Onken, Chem. Eng. Sci. 37, 1635 (1982) and H. K. Tsao and D. L. Koch, Phys. Fluids A 6, 2591 (1994). $\odot 1996$ Academic Press, Inc. Key Words: bubbles; coalescence; nonionic surfactants; high R eynolds number.
\end{abstract}

\section{INTRODUCTION}

The bouncing and coalescence of two bubbles in a liquid are processes of great importance in two-phase flows. The coalescence of a pair of bubbles increases bubble size and decreases the total interfacial area, which changes the velocity of rise and mass transfer. In this paper, interaction between relatively large bubbles (of order $1 \mathrm{~mm}$ ) in water will be studied. In the approach process between these bubbles inertia plays a dominant role (e.g., $(1-6)$ ), in contrast to the approach of very small bubbles or drops in viscous liquids where inertia is negligible and thinning can be described by Stokes flow (e.g., $(7,8))$.

In pure water the thinning of inertia-dominated bubbles, i.e., bubbles with high Reynolds number $(\operatorname{Re}=2 \rho U R / \mu)$, is studied by several authors $(1-6)$. It was found that bubbles coalesce at sufficiently low Weber number ( We $=\rho V^{2} R /$ $\sigma$ ), so for a given bubble size and liquid bubbles bounce if the approach velocity exceeds a critical value.

Water is only free from impurities when special precautions are taken. If surfactants are present in the water, they adsorb on the bubble surface. The influence of these surfactants on bubbles rising through contaminated water are twofold: decrease of the velocity of rise of a bubble (9), and above a critical concentration of surfactants coalescence is inhibited $(5,6,10-13)$.

The coalescence inhibition by added surfactants is a process which is not well understood. Several models based on

\footnotetext{
${ }^{1}$ Present address: Phillips Research, Prof. Holstlaan 4, 5656 AA Eindhoven, The Netherlands.
}

a surface tension gradient caused by film stretching have been proposed (14-17). None of these models, however, can explain the experimental results of bubble coalescence in aqueous solutions of nonionic surfactants $(6,10-13)$.

In this paper we consider the electric effects of nonionic surfactants (e.g., $n$-alcohols) on film thinning. The molecules of these surfactants have a dipole moment and exert a repelling force on film thinning. Keitel and Onken (11) and more recently Tsao and Koch (18) suggest that an electric force of nonionic surfactants may be the cause of coalescence inhibition of inertia-driven bubbles. Sagert and Quinn (19) have calculated the effect of electric double-layer forces in solutions of electrolytes. They concluded that for electrolytes electric double layers have negligible influence. For $n$-alcohols electric effects can be of importance, according to these authors. Therefore, the repelling force due to electric dipoles of $n$-alcohols has been calculated and is included in a simple model to predict coalescence or bouncing of bubbles. The influence of electric dipoles of $n$-alcohols is, to the best knowledge of the author, not yet theoretically investigated. The results can be extended to other nonionic surfactants.

During thinning, three parameters are changing (Fig. 1): the radius of contact $a$, the velocity of the bubble center, and the film thickness $h$. We shall first obtain expressions for the forces working on the bubbles during approach. With these forces a thinning equation and an equation of motion for the bubble center are derived with which the film radius, the velocity of the bubble center, and the film thickness can be calculated during thinning. From our calculations it can be concluded that electric dipoles cannot be the cause of coalescence inhibition.

\section{REPELLING FORCE DUE TO DIPOLE MOMENTS}

If bubbles are formed in an aqueous solution of $n$-alcohols there will be diffusion of surfactant molecules to the bubble surface. As a simplification all $n$-alcohols are denoted here as surfactants because they influence the surface tension. These molecules are so-called head-tail molecules, of which the polar head is hydrophilic and the apolar tail is hydrophobic. The dipole moments of surfactant molecules on two 

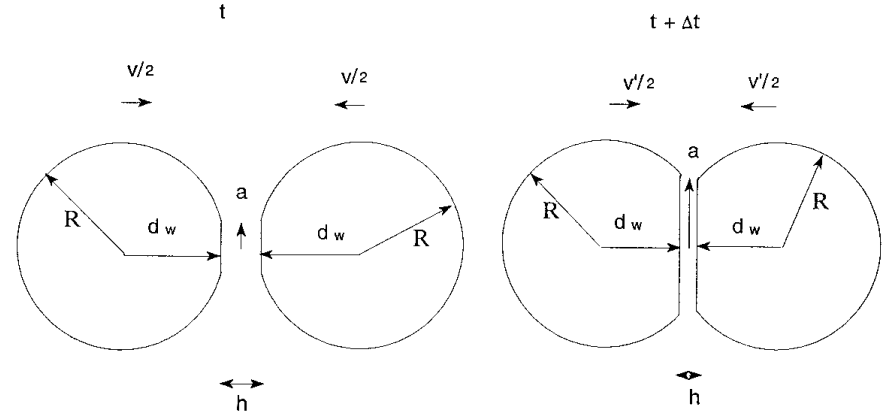

FIG. 1. Horizontal bubble approach.

approaching bubbles are in opposite direction and therefore exert a repelling force on each other. Here the configuration of Fig. 2 is used, and in doing so the repelling dipole force is overestimated. In reality molecules will not be ordered in this way; by dynamic effects the molecules will continuously alter their position, and for dilute solutions the tails of the molecules will not stick in the air but will lie on the surface (20). The electric dipole force will, like the van der Waals force, only play a role at small film thicknesses $\left(\sim 10^{-7} \mathrm{~m}\right)$.

In order to calculate the forces working on the bubble the following assumptions are made: (1) $\Gamma$ is constant and in equilibrium on the bubble surface. (2) The bubbles approach each other as sketched in Fig. 1. (3) The film between the bubbles is parallel sided and the radius of contact $a$ is assumed small with respect to $R$ (Fig. 1).

ad 1. By assuming a constant surface concentration, convection and diffusion are neglected in the mass balance of surfactants on the bubble surface.

ad 2. Kok $(4,5)$ showed that in inertia-controlled motion, two spherical bubbles approach each other as sketched in Fig. 1.

ad 3. The assumption of a plan parallel film is not fully correct; in reality the film will, by inertia, develop a dimple (2).

With these assumptions the repelling force between the bubbles due to dipole moments can be estimated from the
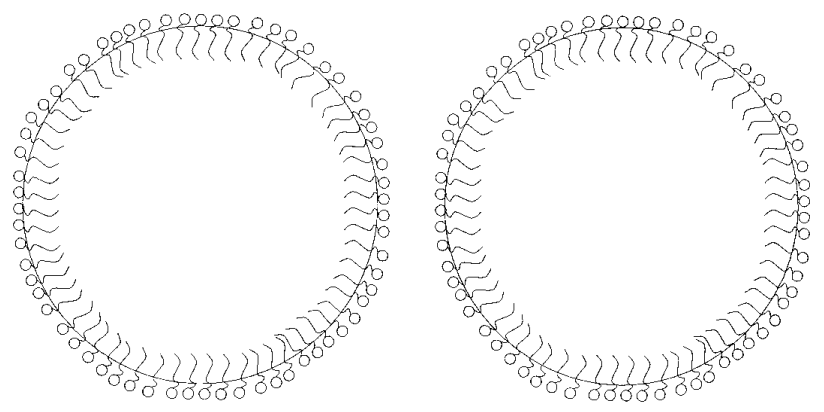

FIG. 2. Surfactant molecules on the bubble surface.

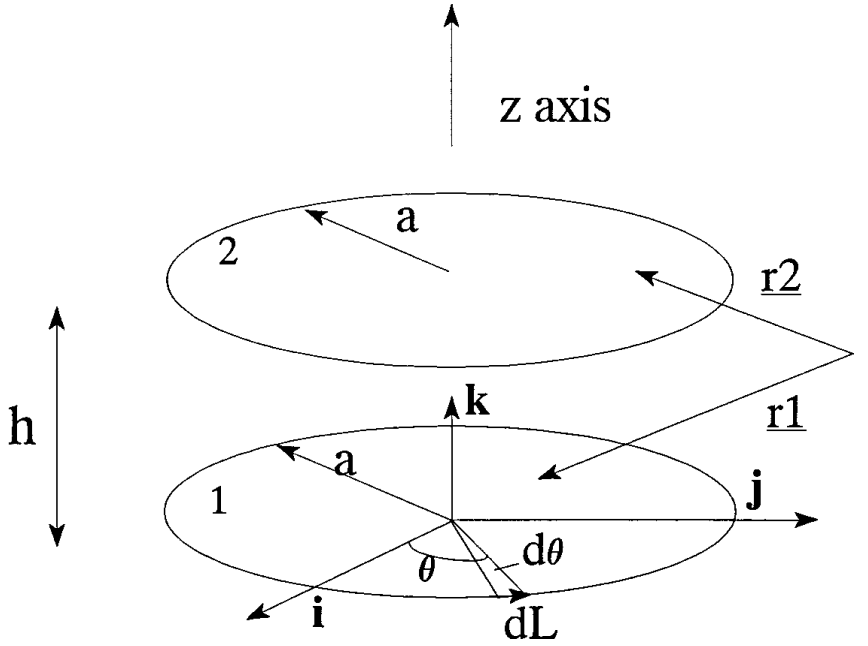

FIG. 3. Model of circular disks.

repelling force between two infinitely thin circular disks with a constant dipole moment per area. This simplification can be made because $h$ is orders of magnitude larger than the size of the surfactant molecules. In the experiments the concentrations of surfactants are so low that the influence on bulk properties of the liquid between the disks can be neglected.

We consider the problem of two disks, both with radius $a$, a constant $\tau$, and distance $h$ between the disks, with the symmetry axis in the $z$ direction (Fig. 3 ).

The potential $\phi$ of an electric dipole is (21)

$$
\phi=\frac{\underline{p} \cdot\left(\underline{r_{2}}-\underline{r_{1}}\right)}{\left|\underline{r_{2}}-\underline{r_{1}}\right|^{3}} \frac{1}{4 \pi \epsilon_{0} \epsilon_{r}} .
$$

The potential $\phi$ in a point of disk 2 , due to disk 1 , is

$$
\phi=\frac{\tau}{4 \pi \epsilon_{0} \epsilon_{r}} \int_{S} \frac{d S_{1}}{\phi} \cdot \nabla_{2}\left(\frac{1}{\left|\underline{r_{2}}-\underline{r_{1}}\right|}\right),
$$

where the $\nabla_{2}$ operator works on $\underline{r}_{2}$. The electric field in a point of disk 2 , due to disk 1 , is

$$
\underline{E}=\frac{-\tau}{4 \pi \epsilon_{0} \epsilon_{r}} \int_{S} \nabla_{2}\left(\underline{d S_{1}} \cdot \nabla_{2}\left(\frac{1}{\left|\underline{r_{2}}-\underline{r_{1}}\right|}\right)\right) .
$$

This can be transformed to

$$
\underline{E}=\frac{-\tau}{4 \pi \epsilon_{0} \epsilon_{r}} \int_{S}\left(\underline{d S_{1}} \cdot \nabla_{2}\right) \nabla_{2}\left(\frac{1}{\left|\underline{r_{2}}-\underline{r_{1}}\right|}\right) .
$$

With a variation on Stokes' theorem, 


$$
\begin{aligned}
\int_{S}\left(\underline{d S_{1}} \cdot \nabla\right) \underline{f}=\oint \underline{d l} \times \underline{f} & +\int_{S}(\nabla \cdot \underline{f}) \underline{d S_{1}} \\
& +\int_{S}(\nabla \times \underline{f}) \times \underline{d S_{1}},
\end{aligned}
$$

we find

$$
\underline{E}=\frac{-\tau}{4 \pi \epsilon_{0} \epsilon_{r}} \oint \underline{d L_{1}} \times \nabla_{2}\left(\frac{1}{\left|\underline{r_{2}}-\underline{r_{1}}\right|}\right) .
$$

The force exerted by disk 1 on disk 2 is (21) $\tau \int_{A}\left(\underline{d A_{2}} \cdot \nabla_{2}\right) \underline{E}$, in which the dipole moment of disk 2 is in the opposite direction of disk 1. Again [5] is used, and because both the curl and the divergence of $\underline{E}$ are vanishing we find that

$$
\underline{F_{2}}=\frac{\tau^{2}}{4 \pi \epsilon_{0} \epsilon_{r}} \oint \oint \underline{d L_{2}}
$$

$$
\times\left(\underline{d L_{1}} \times \nabla_{2}\left(\frac{1}{\left|\underline{r_{2}}-\underline{r_{1}}\right|}\right)\right) .
$$

The calculation of the force from two surface integrals is reduced to two path integrals. By using Stokes theorem it can be proved that this force is, as expected, antisymmetric:

$$
\underline{F_{2}}=-\underline{F_{1}}=\frac{\tau^{2}}{4 \pi \epsilon_{0} \epsilon_{r}} \oint \oint \underline{d L_{1}} \cdot \underline{d L_{2}} \frac{\underline{r_{2}}-\underline{r_{1}}}{\left|\underline{r_{2}}-\underline{r_{1}}\right|^{3}} .
$$

The integrals in the $i$ and in the $j$ direction are both vanishing; the force has only a component in the $k$ direction,

$$
\begin{aligned}
F_{2}= & \frac{\tau^{2}}{4 \pi \epsilon_{0} \epsilon_{r}} \int_{0}^{2 \pi} \int_{0}^{2 \pi} \\
& \times \frac{h a^{2} \cos \left(\theta_{2}-\theta_{1}\right)}{\left[2 a^{2}-2 a^{2} \cos \left(\theta_{2}-\theta_{1}\right)+h^{2}\right]^{3 / 2}} d \theta_{1} d \theta_{2} .
\end{aligned}
$$

Taking $\theta_{2}-\theta_{1}=\xi$ gives

$$
\begin{aligned}
F_{2}=\frac{\tau^{2}}{4 \pi \epsilon_{0} \epsilon_{r}} \int_{0}^{2 \pi} d \theta_{1} & \int_{-\theta_{1}}^{2 \pi-\theta_{1}} \\
& \times \frac{h a^{2} \cos \xi}{\left[2 a^{2}-2 a^{2} \cos \xi+h^{2}\right]^{3 / 2}} d \xi .
\end{aligned}
$$

Changing the sequence of integration gives for the integral

$$
\begin{aligned}
\frac{\tau^{2} h a^{2}}{2 \epsilon_{0} \epsilon_{r}} \int_{0}^{2 \pi} \frac{\cos \xi}{\left[2 a^{2}-2 a^{2} \cos \xi+h^{2}\right]^{3 / 2}} d \xi-\frac{\tau^{2} h a^{2}}{4 \pi \epsilon_{0} \epsilon_{r}} \\
\quad \times \int_{0}^{2 \pi} \frac{\xi \cos \xi}{\left[2 a^{2}-2 a^{2} \cos \xi+h^{2}\right]^{3 / 2}} d \xi .
\end{aligned}
$$

By using the following theorem (22)

$$
\begin{aligned}
\int_{0}^{n \pi} f(\sin x) x d x= & n^{2} \pi \int_{0}^{\pi / 2} f(\sin x) d x, \\
n & =0,1,2 \cdots \text { if } f(y)=f(-y),
\end{aligned}
$$

it follows that

$$
F_{2}=\frac{\tau^{2}}{4 \epsilon_{0} \epsilon_{r}} \int_{0}^{2 \pi} \frac{h a^{2} \cos \xi}{\left[2 a^{2}-2 a^{2} \cos \xi+h^{2}\right]^{3 / 2}} d \xi
$$

Taking $\gamma=\theta / 2$ we find that

$$
\begin{aligned}
F_{2}= & \frac{\tau^{2} h a^{2}}{2 \epsilon_{0} \epsilon_{r}\left[4 a^{2}+h^{2}\right]^{3 / 2}} \\
& \times \int_{0}^{\pi} \frac{2 \cos ^{2} \gamma-1}{\left[1-\left[4 a^{2} /\left(4 a^{2}+h^{2}\right)\right] \cos ^{2} \gamma\right]^{3 / 2}} d \gamma .
\end{aligned}
$$

This is a standard integral (e.g., (22)), giving

$$
\begin{aligned}
F_{2}=\frac{\tau^{2} \pi a^{2} h}{2 \epsilon_{0} \epsilon_{r}\left[4 a^{2}+h^{2}\right]^{3 / 2}} & {\left[{ }_{2} F_{1}\left(\frac{3}{2}, \frac{3}{2} ; 2 ; \frac{4 a^{2}}{4 a^{2}+h^{2}}\right)\right.} \\
& \left.-{ }_{2} F_{1}\left(\frac{1}{2}, \frac{3}{2} ; 1 ; \frac{4 a^{2}}{4 a^{2}+h^{2}}\right)\right] .
\end{aligned}
$$

In the experiments $h / 2 a \ll 1$, which we discuss later; therefore, the following theorem can be used (23):

If $a+b>c \geqslant b>0$ and $x \rightarrow 1$ then

$$
{ }_{2} F_{1}(a, b ; c ; x) \rightarrow \frac{\Gamma(c) \Gamma(a+b-c)}{\Gamma(a) \Gamma(b)}(1-x)^{c-a-b} .
$$

So

$$
F_{2}=\frac{\tau^{2} \pi a^{2} h}{\epsilon_{0} \epsilon_{r}\left[4 a^{2}+h^{2}\right]^{3 / 2}} \frac{1}{\pi}\left(1-\frac{4 a^{2}}{4 a^{2}+h^{2}}\right)^{-1} .
$$

With $h^{2} \ll a^{2}$ this can be simplified to

$$
F_{2}=\frac{\tau^{2} a}{2 \epsilon_{0} \epsilon_{r} h} .
$$


This is the force disk 1 exerts on disk 2, which is in the positive $z$ direction and therefore repelling.

The dipole moment per area, $\tau$, is given by

$$
\tau=p \Gamma N_{\mathrm{a}} .
$$

The surface concentration is determined with the GibbsDuhem relation (20), which holds for dilute solutions:

$$
\Gamma=\frac{-c}{R_{\mathrm{g}} T}\left(\frac{d \sigma}{d c}\right)_{T} .
$$

In the experiments dilute solutions of $n$-alcohols in water are used. With the Von Szyskowski equation (24), an expression is found for the equilibrium surface tension as a function of the surfactant concentration:

$$
\sigma-\sigma_{0}=-R_{\mathrm{g}} T \Gamma_{\infty} \ln \left(1+\frac{c}{a^{\prime}}\right) .
$$

For different $n$-alcohols $\Gamma_{\infty}$ is nearly constant, but $a^{\prime}$ is changing drastically with the number of $\mathrm{C}$ atoms in the $n$ alcohol. Using [17] and [18] gives for the equilibrium surface concentration $\Gamma$

$$
\Gamma=\frac{\Gamma_{\infty} c}{a^{\prime}+c} .
$$

Substituting [16] and [19] in [15] gives

$$
F_{\text {dip }}=\frac{1}{2 \epsilon_{0} \epsilon_{r}} \frac{\left(\Gamma_{\infty} c\right)^{2}}{\left(a^{\prime}+c\right)^{2}} p^{2} N_{\mathrm{a}}^{2} \frac{a}{h} .
$$

For small surface concentrations it can be shown (6) that the dipole moment of a $n$-alcohol molecule on the bubble surface is nearly equal to the dipole moment of water, giving $p=6.1 \times 10^{-30} \mathrm{C} \mathrm{m}(25)$.

\section{DEFORMATION AND VAN DER WAALS FORCE}

When during approach bubbles become close, the pressure in the liquid film will, due to inertia, increase sharply. This pressure will deform and exert a repelling force on the bubbles. Here it is assumed that the bubbles only deform if $d_{\mathrm{w}}$ $<R$ (Fig. 1). With a parallel-sided film assumption and the normal stress condition on the bubble surface, $P_{\mathrm{g}}=P_{1}+$ $2 \sigma / R_{\mathrm{c}}$, the repelling force becomes

$$
F_{\text {def }}=\frac{2 \pi \sigma a^{2}}{R}
$$

Note that the pressure in the bubble and hence the bubble volume remains constant. The deformation of the bubble is caused by the increase of the liquid pressure due to the stagnation point in the film center.

The equation for determining the film radius is easily found by elementary geometry

$$
a^{2}=2 R\left(R-d_{\mathrm{w}}\right),
$$

where we have used $d_{\mathrm{w}} \approx R$. With the constant volume constraint the deformation causes an increase of $R$. Elementary geometry shows however that this increase is for small $a$ negligible, hence $R$ is constant. So, if this position of the bubble center is known, $d_{\mathrm{w}}$ and $a$ can be calculated.

The attractive unretarded van der Waals force is valid if $h$ is less than about $3 \times 10^{-8} \mathrm{~m}(1,20)$ and is

$$
F_{\mathrm{vdW}}=\frac{-A}{6 \pi h^{3}} \pi a^{2}
$$

For water $A=2.4 \times 10^{-20} \mathrm{~kg} \mathrm{~m}^{2} \mathrm{~s}^{-2}(20)$.

If $h$ is larger than $3 \times 10^{-8} \mathrm{~m}$, the retarded van der Waals force must be used,

$$
F_{\mathrm{vdW}}=\frac{-B}{h^{4}} \pi a^{2}
$$

where $B$ is a constant, of order $10^{-28} \mathrm{~kg} \mathrm{~m}^{3} \mathrm{~s}^{-2}(26)$. Here Eq. [24] is used because $h$ exceeds $3 \times 10^{-8} \mathrm{~m}$, with $B$ taken as $1 \times 10^{-28} \mathrm{~kg} \mathrm{~m}^{3} \mathrm{~s}^{-2}$.

\section{THE THINNING EQUATION}

Now that all the forces appearing in our model have been calculated, the thinning equation will be derived. This equation is based on Chesters (1) where also a plan parallel film was assumed. Using continuity and the Navier-Stokes equation, the thinning equation [31] of Chesters' paper can be derived

$$
\frac{d^{2} H}{d t^{2}}+\frac{4 \mu}{\rho a^{2}} \frac{d H}{d t}+\frac{2 P_{0}}{\rho a^{2}}=0
$$

In Chesters (1) Eq. [25] was derived for a pure liquid; therefore the velocity across the film was constant. Because here the surfactant concentration is assumed to be constant, the surface remains completely mobile $(27,28)$ and therefore vorticity generation is zero, resulting in a constant velocity across the film. The surface cannot become fully mobile by a gradient in surface concentration; however, this effect will not be considered here. 
The balancing of forces on an element at the center of the film gives

$$
P_{0}=4 \mu\left(\frac{d H}{d t}\right)+\frac{2 \sigma}{R}+\frac{B}{h^{4}}-\frac{F_{\mathrm{dip}}}{\pi a^{2}}
$$

The first term on the right side is due to the normal stress at the film center; the other terms are the pressure due to deformation (which increase thinning of the liquid film), the van der Waals pressure, and the dipole pressure, where $F_{\text {dip }}$ is given by [20].

Substituting [26] in [25] gives

$$
\frac{d^{2} H}{d t^{2}}+\frac{12 \mu}{\rho a^{2}} \frac{d H}{d t}+\frac{4 \sigma}{\rho a^{2} R}+\frac{2 B}{\rho a^{2} h^{4}}-\frac{2 F_{\mathrm{dip}}}{\rho \pi a^{4}}=0 .
$$

For the initial value of the film thickness we follow Chesters and Hofman (2), $h_{0}=\left(4 \rho V^{2} R^{2}\right) / \sigma$. The initial value of the contact radius $a$ is not zero but chosen very small, $a_{0}=1$ $\times 10^{-7} \mathrm{~m}$. Our results show that small variations of $h_{0}$ and $a_{0}$ have little influence on the results.

\section{EQUATION OF MOTION FOR APPROACHING BUBBLES}

In the coalescence models given in the literature the only aim was to derive an equation for the thinning of the film between two bubbles. A constant velocity of approach is assumed in these models. However during approach forces are exerted on each bubble. In our model these forces are the repelling force, due to dipoles and bubble deformation, $F_{\text {dip }}$ and $F_{\text {def }}$, respectively, and the attracting van der Waals force, $F_{\mathrm{vdw}}$.

The inertia of the bubble is zero but the liquid displaced by the bubble has an apparent mass, expressed by $M_{0}$, the added mass. The added mass of a bubble, as sketched in Fig. 1, is (29)

$$
M_{0}=0.36 \cdot \frac{4}{3} \pi R^{3} \rho
$$

This added mass is smaller than that of a single rising spherical bubble $\left(0.5 \cdot \frac{4}{3} \pi R^{3} \rho\right)$ which is caused by the position and the relative motion of the bubbles. Using the concept of added mass the equation of motion for the center of mass becomes

$$
\frac{d\left(M_{0} \underline{u}\right)}{d t}=\underline{F}_{\mathrm{dip}}+\underline{F}_{\mathrm{def}}+\underline{F}_{\mathrm{vdW}},
$$

where $\underline{u}$ is the velocity of the center of mass,

$$
\frac{d d_{\mathrm{w}}}{d t}=-u
$$

Using [20], [21], and [23], [29] becomes

$$
\begin{aligned}
\left.\frac{d\left(M_{0} \underline{u}\right)}{d t}=\frac{1}{2 \epsilon_{0} \epsilon_{r}} \frac{(}{\left(a_{\infty} c\right)^{2}}+c\right)^{2} & \\
& \quad \times p^{2} N_{\mathrm{a}}^{2} \frac{a}{h}+\frac{2 \sigma \pi a^{2}}{R}-\frac{B}{h^{4}} \cdot \pi a^{2},
\end{aligned}
$$

with initial conditions $u=1 / 2 V$ and $d_{\mathrm{w}}=R$.

Our model is only valid up to a certain critical film thickness. This critical thickness for coalescence depends on the Hamaker constant. In Chesters (1) the critical thickness is estimated to be a few hundred angström. In Doubliez (30) the critical thickness for a bubble rising to a free surface was measured. A minimal thickness was found to be $800 \AA$. Here the critical thickness is estimated to be $3 \times 10^{-8} \mathrm{~m}$, which is on the same order as the values given by Chesters (1) and Doubliez (30). Choosing any other reasonable value shows no effect on the results discussed in the next section.

A criterium for coalescence or bouncing is:

- If the thinning is stopped $(d h / d t=0)$ before a critical thickness is reached the bubbles will bounce.

- If the film between the bubbles has reached a critical thickness and the thinning of the film has not been reduced to zero, the bubbles will coalesce.

The aim is now to determine the critical concentration of the $n$-alcohol which will prevent coalescence. This is done by solving [22], [27], and [31] with the initial conditions by a standard fourth-order Runge-Kutta scheme.

\section{COMPARISON WITH EXPERIMENTS AND DISCUSSION}

The experiments of Keitel and Onken (10) are used to verify our model. In these experiments coalescence inhibition of bubbles $\left(R \sim 5 \times 10^{-4} \mathrm{~m}\right)$ by $n$-alcohols is studied in a bubble column where bubbles rise under buoyancy. These authors concluded that, above a critical concentra-

TABLE 1

Experimental and Theoretical Critical Concentrations for R ising Bubbles

\begin{tabular}{lcccc}
\hline$n$-alcohol & $\begin{array}{c}C_{\text {model }} \\
\left(\mathrm{mol} / \mathrm{m}^{3}\right)\end{array}$ & $\begin{array}{c}C_{\text {K\&O }} \\
\left(\mathrm{mol} / \mathrm{m}^{3}\right)\end{array}$ & $\begin{array}{c}a^{\prime} \\
\left(\mathrm{mol} / \mathrm{m}^{3}\right)\end{array}$ & $\begin{array}{c}\Gamma_{\infty} \\
\left(\mathrm{mol} / \mathrm{m}^{2}\right)\end{array}$ \\
\hline Butanol & - & $4 \times 10^{-2}$ & $4.7 \times 10^{1}$ & $6 \times 10^{-6}$ \\
Octanol & - & $3 \times 10^{-3}$ & $2.1 \times 10^{-1}$ & $6 \times 10^{-6}$ \\
\hline
\end{tabular}




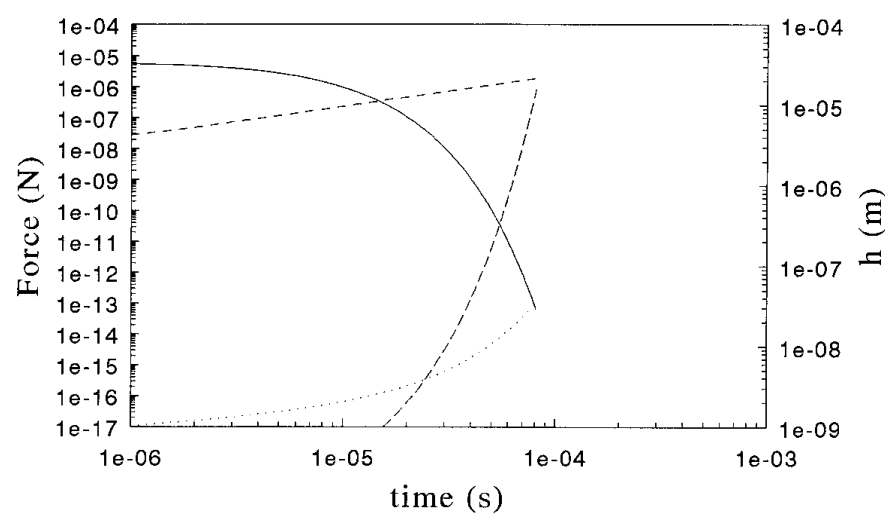

FIG. 4. Forces working in the liquid film for rising bubbles in an aqueous solution of octanol; $c=3 \times 10^{-3} \mathrm{~mol} / \mathrm{m}^{3}, V=0.05 \mathrm{~m} / \mathrm{s}$. - , $h ;---, F_{\mathrm{vdW}} ;---, F_{\text {def }} ; \cdots, F_{\text {dip }}$.

tion of the $n$-alcohol, coalescence was inhibited. The approach velocity in the experiments is unknown. This velocity was estimated using van Wijngaarden's (31) theory and experiments (6) of bubble pairs. The approach velocity of bubble pairs just before "contact" depends on the initial separation between the bubble centers. At the maximum separation where the bubbles attract each other $(\approx 8 R)$ it was found that the approach velocity is $\approx 0.5 U$, where $U$ is the vertical rise velocity of the bubbles. With $U \approx 0.20 \mathrm{~m} / \mathrm{s}$ for bubbles with $R=5 \times 10^{-4} \mathrm{~m}$ this gives $V_{\max }=0.10 \mathrm{~m} / \mathrm{s}$. However if the initial separation between the bubbles is smaller the approach velocity decreases. Therefore we have estimated $V \approx 0.05 \mathrm{~m} / \mathrm{s}$ for the calculations of Fig. 4. Changing to the maximum approach velocity gives no other results.

From Table 1 it follows that our model, which overestimates the influence of the repelling dipole force, gives for both alcohols no critical concentration to prevent coalescence. Even the maximum surface concentration $\Gamma_{\infty}$ gives a repelling dipole force which is orders of magnitude too small to inhibit coalescence. Comparison with other $n$-alcohols gives similar results.

In Fig. 4 the different forces, calculated with [20], [21], and [24], are plotted for a typical concentration used in the experiment. It is obvious that the electric dipole force is not sufficient to prevent coalescence.

The previously discussed experiments were rising bubbles in a bubble column. The experiments $(10,12,13)$ all deal with two bubbles, each growing on a capillary. An approximation for the approach velocity $V$ follows from a mass balance

$$
V=\frac{2}{3} f R
$$

In the equation of motion [29] an extra term is obtained because the added mass is not constant in time. This extra term is $\underline{u} M_{0} f$ and negligible compared to the other terms. Also in these experiments coalescence is prevented at concentrations which are orders of magnitude smaller than this model predicts.

In our model the calculations were based on a plan-parallel film, while in reality a dimple develops. In doing so the thinning time is overestimated (2), which overestimates the repelling dipole force.

The initial condition for the contact area is here taken as $10^{-7} \mathrm{~m}$, but this contact area increases very fast during thinning (at the end of thinning $a$ is of the order of $10^{-5} \mathrm{~m}$ ), while $h$ decreases very fast, as can be seen from Fig. 4. So $h / 2 a \ll 1$ during almost the complete thinning process and $a \ll R$.

Changing the critical film thickness and the approach velocity to any other reasonable value has no influence on the result.

All assumptions overestimate the repelling dipole force; still the critical concentration calculated is orders of magnitude larger than the experimental value. So it can be concluded that the repelling dipole force cannot be the cause of the bouncing of bubbles in aqueous solutions of $n$-alcohols. There must be a force, not taken into account in our model, which is much larger than the repelling dipole force and responsible for the coalescence inhibition.

The suggestion here is that this force is caused by a surface tension gradient, which will be only qualitatively discussed here. In the Introduction some coalescence models have been already mentioned. All these are based on a surface tension gradient, caused by film stretching; i.e., in all models a fully immobile surface is assumed. This assumption is, however, too rigorous. When there are no surfactants present, the liquid in the film can slip at the bubble surface during thinning and no film stretching occurs. At the presence of very small surfactant concentrations the surface will first be partly mobile and the mobility decreases with increasing surfactant concentration. The immobile surface assumption therefore overestimates the surface tension gradient, hence underestimating the critical concentration. The reduction in surface mobility is due to a concentration gradient in the film, which is caused by a convective accumulation of surfactant at the rim of the film, where the flow diverges enormously. This mechanism also occurs in flows of bubbles in capillaries (e.g., 27, 32, 33) and the created surface tension gradient may, at sufficient surfactant concentration, stop film thinning.

\section{APPENDIX: NOMENCLATURE}

$\begin{array}{ll}A & \text { Hamaker constant, } \mathrm{kg} \mathrm{m}^{2} \mathrm{~s}^{-2} \\ a & \text { Radius of contact, } \mathrm{m} \\ a^{\prime} & \text { Langmuir constant, mol m} \\ B & \text { Retarded van der Waals force constant, } \mathrm{kg} \mathrm{m}^{3} \mathrm{~s}^{-2}\end{array}$


$C_{\text {model }}$ Critical concentration model, $\mathrm{mol} \mathrm{m}^{-3}$

$C_{\mathrm{K} \& \mathrm{O}}$ Experimental critical concentration by Keitel and Onken (10), $\mathrm{mol} \mathrm{m}^{-3}$

c Concentration, mol m $\mathrm{m}^{-3}$

$d_{\mathrm{w}} \quad$ Distance bubble center to disk center (Fig. 1), $\mathrm{m}$

$E \quad$ External electric field, $\mathrm{N} \cdot \mathrm{C}^{-1}$

$F_{\text {def }} \quad$ Deformation force, $\mathrm{N}$

$F_{\text {dip }} \quad$ Dipole force, $\mathrm{N}$

$F_{i} \quad$ Force exerted by disk $(3-i)$ on disk $i, \mathrm{~N}$

$F_{\text {vdW }}$ van der Waals force, $\mathrm{N}$

$f \quad$ Bubble generation frequency, $\mathrm{s}^{-1}$

$h \quad$ Film thickness, $m$

H $\quad 1 / 2 \ln (h)$,

$L_{i} \quad$ Line element disk $i, \mathrm{~m}$

$N_{\mathrm{a}} \quad$ Avogadro's number, $\mathrm{mol}^{-1}$

$P_{\mathrm{g}} \quad$ Pressure in the bubble, $\mathrm{N} \mathrm{m}^{-2}$

$P_{1} \quad$ Pressure in the liquid, $\mathrm{N} \mathrm{m}^{-2}$

$P_{0} \quad$ Pressure in the center of the film with respect to $P_{\infty}$, $\mathrm{N} \mathrm{m}^{-2}$

$P_{\infty} \quad$ Undisturbed pressure, $\mathrm{N} \mathrm{m}^{-2}$

$p \quad$ Dipole moment of one molecule, $\mathrm{C} \mathrm{m}$

$R \quad$ Radius of the bubble, $\mathrm{m}$

$R_{\mathrm{c}} \quad$ Radius of curvature, $\mathrm{m}$

$R_{\mathrm{g}} \quad$ Gas constant, $\mathrm{J} \mathrm{mol}^{-1} \mathrm{~K}^{-1}$

$r \quad$ Radial distance in the film, $\mathrm{m}$

$r_{i} \quad$ Vector from origin to disk $i, \mathrm{~m}$

$S_{i} \quad$ Area disk $i, \mathrm{~m}^{2}$

$T \quad$ Temperature, $\mathrm{K}$

$t \quad$ Time, s

$u \quad$ Velocity center of mass, $\mathrm{m} \mathrm{s}^{-1}$

$U \quad$ Vertical velocity of rise of the bubble, $\mathrm{m} \mathrm{s}^{-1}$

$V \quad$ Velocity of approach, $\mathrm{m} \mathrm{s}^{-1}$

$\Gamma \quad$ Surface concentration, $\mathrm{mol} \mathrm{m} \mathrm{m}^{-2}$

$\Gamma_{\infty} \quad$ Saturation surface concentration, $\mathrm{mol} \mathrm{m} \mathrm{m}^{-2}$

$\epsilon_{0} \quad$ Permitivity of free space, $\mathrm{C}^{2} \mathrm{~m}^{-2} \mathrm{~N}^{-1}$

$\epsilon_{r} \quad$ Dielectric constant

$\phi \quad$ Electric potential, $\mathrm{N} \mathrm{C}^{-1} \mathrm{~m}^{-1}$

$\theta_{i} \quad$ Angle with $i$ axis on disk 1, rad

$\mu \quad$ Dynamic viscosity, $\mathrm{kg} \mathrm{m} \mathrm{s}^{-2}$

$\rho \quad$ Density, $\mathrm{kg} \mathrm{m}^{-3}$

$\sigma \quad$ Surface tension, $\mathrm{N} \mathrm{m}^{-1}$

$\sigma_{0} \quad$ Surface tension of "clean" water, $\mathrm{N} \mathrm{m}^{-1}$

$\tau \quad$ Dipole moment per area, $\mathrm{C} \mathrm{m}^{-1}$

\section{ACK NOWLEDGMENT}

I thank Professor L. van Wijngaarden for many helpful discussions and a continuous interest in this work.

\section{REFERENCES}

1. Chesters, A. K., Int. J. Multiphase Flow 2, 191 (1975).

2. Chesters, A. K., and Hofman, G., Appl. Sci. Res. 38, 353 (1982).

3. Chesters, A. K., Inst. Chem. Eng. 69, 259 (1991).

4. Kok, J. B. W., Eur. J. Mech. B/Fluids 4, 515 (1993).

5. Kok, J. B. W., Eur. J. Mech. B/Fluids 4, 541 (1993).

6. Duineveld, P. C., "Bouncing and Coalescence of Two Bubbles in Water." Ph.D. thesis, University of Twente, 1994.

7. Dongming, L., J. Colloid Interface Sci. 163, 108 (1994).

8. Manga, M., and Stone, H. A., J. Fluid Mech. 256, 647 (1993).

9. Bel Fdhila, R., and Duineveld, P. C., Phys. Fluids A 8, 310 (1996).

10. Drogaris, G., and Weiland, P., Chem. Eng. Sci. 38, 1501 (1983).

11. Keitel, G., and Onken, U., Chem. Eng. Sci. 37, 1635 (1982).

12. Kim, J. W., and Lee, W. K., J. Colloid Interface Sci. 123, 303 (1988).

13. Yang, Y. M., and Maa, J. R., J. Colloid Interface Sci. 98, 120 (1984).

14. Andrews, S. P. S., Inst. Chem. Eng. Symp. Ser. Distillation, 73 (1960).

15. Marruci, G., Chem. Eng. Sci. 24, 975 (1969).

16. Oolman, T. O., and Blanch, H. W., Chem. Eng. Commun. 43, 237 (1986).

17. Prince, J. P., and Blanch, H. W., AIChE. J. 36, 1425 (1990).

18. Tsao, H. K., and Koch, D. L., Phys. Fluids A 6, 2591 (1994).

19. Sagert, N. H., and Quinn, M. J., Chem. Eng. Sci. 33, 1087 (1978).

20. Hiemenz, P. C., 'Principles of Colloid and Surface Chemistry.' Dekker, New York, 1989.

21. Panofsky, W. K. H., and Phillips, M. B., "Classical Electricity and Magnetism.', Weshley, 1956.

22. Gröbner, W., and Hofreiter, N., "Inegraltafel, Bestimmte Integrale." Springer-Verlag, Wien, 1966.

23. Morse, P. M., and Feshbach, H., "Methods of Theoretical Physics," p. 388. McGraw-Hill, New York, 1983.

24. Bleys, G., and Joos, P., J. Phys. Chem. 89, 1027 (1985).

25. Böttcher, C. J. F., 'Theory of Electric Polarization.” Elsevier, Amsterdam, 1973.

26. Israelachvili, J. N., and Tabor, D., in "Progress in Surface and Membrane Science', (J. F. Danielli, M. O. Rosenberg, and D. A. Cadenhead, Eds.), Vol. 7, p. 1. Academic Press, New York, 1973.

27. Stebe, K. J., Lin, S. Y., and Maldarelli, C., Phys. Fluids A 3, 3 (1991).

28. Stebe, K. J., and Maldarelli, C., J. Colloid Interface Sci. 163, 177 (1994).

29. Miloh, T., J. Eng. Math. 11, 349 (1977).

30. Doubliez, L., Int. J. Multiphase Flow 17, 783 (1991).

31. Wijngaarden, L. van, J. Fluid Mech. 251, 55 (1993).

32. Ratulowski, J., and Chang, H. C., J. Fluid Mech. 210, 303 (1990).

33. Park, C. W., Phys. Fluids A 4, 2335 (1992). 